Executive Director conference was financially secure. Thanks to the Ottawa Valley section, Kathryn Buchanan and Keith Forbes for organizing the silent auction, and Ron Morrison for coordinating the audiovisual requirements for the speakers and meetings. Even companions were brought into the meeting this year, and Julie Nordin and Mary Geale, and Karen Haddon all provided assistance. Plus our own Cherry Maxwell and Sue Easterman were on the scene helping. All the Deans/Chairs from our Canadian forestry schools were on hand to host a very successful Alumni breakfast and two Deans, Michel Desseault and Lise Caron, moderated the two technical sessions.

Now having said all that, one must recognize that outside of our two staff, and myself the core group of people organizing the meetings and our Board/Executive are all volunteers. These individuals gave their time, and their employers allowed them to give their time, to make the CIF/IFC 90th annual meeting a success.

So much work goes on behind the scenes of planning annual meetings and conferences, and no written words can explain it all. So one more time, I would like to say thank you to all those involved in the planning and organizing of the CIF/IFC 90th annual meeting, and thank you to our volunteers and members for supporting the Institute for 90 years!
Directrice Exécutive Kathryn Buchanan et Keith Forbes de la section Vallée de l'Outaouais pour l'organisation de l'encan silencieux, ainsi que Ron Morrison pour la supervision des besoins audiovisuels pour les conférenciers et les réunions. Même les conjoints ont été mis à contribution au cours de cette assemblée; Julie Nordin, Mary Geale et Karen Haddon accordèrent leur assistance. Sans oublier Cheery Maxwell et Sue Easterman de notre bureau qui étaient sur place. Enfin tous les doyens ou directeurs des facultés de foresterie du Canada étaient les hôtes du très réussi déjeuner des anciens, et deux doyens, Michel Dessureault et Lise Caron, ont agi à titre de modérateurs lors de deux sessions techniques.

Maintenant que tout cela a été dit, il faut reconnaître qu'à part nos deux employés et moi-même, le principal groupe de personnes en charge de l'organisation de l'assemblée et les membres du conseil d'administration et du comité exécutif sont tous des bénévoles. Ces personnes ont donné de leur temps, et leur employeur, de la disponibilité, pour faire de la 90e assemblée annuelle de l'Institut un succès.

Il y a tant de travail effectué derrière la scène lors de la planification des assemblées annuelles et des conférences qu'aucun texte ne peut expliquer tout cela. Alors, encore une fois, je voudrais remercier tous ceux qui ont été impliqués dans la planification et l'organisation de la 90e assemblée annuelle, et remercier tous les bénévoles et les membres d'avoir appuyer l'Institut pendant 90 ans!

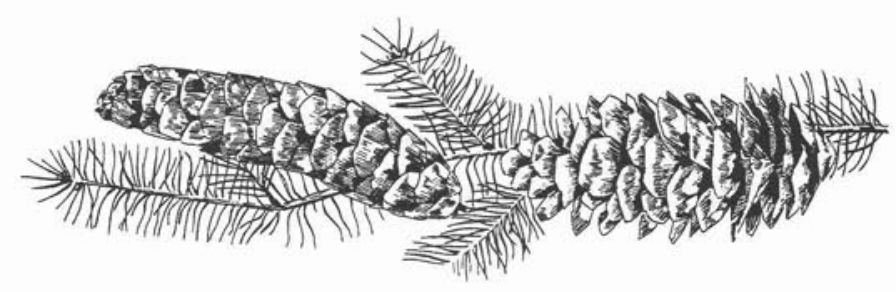

\title{
Individual Sustaining Members 1998-99
}

$\begin{array}{lll}\text { H.W. Beall } & \text { OV } & \text { W. Ross Grinnell } \\ \text { A. Bickerstaff } & \text { OV } & \text { R.D. Hallett } \\ \text { R. Boultbee } & \text { SO } & \text { B.H. Hunt } \\ \text { George Brown } & \text { SO } & \text { C. Allan Jeffrey } \\ \text { Brian Cardwell } & \text { SO } & \text { A.R.C. Jones } \\ \text { J.H. Cayford } & \text { OV } & \text { A. Kabzems } \\ \text { G.W.I. Creighton } & \text { NS } & \text { A.J. Kayll } \\ \text { E.C. Crossin } & \text { VA } & \text { Tom Koftinoff } \\ \text { F. Dottori } & \text { OR } & \text { Nenad Kontic } \\ \text { D.P. Drysdale } & \text { SO } & \text { D.D. Lockhart } \\ \text { William Finlay } & \text { SO } & \text { J.D. MacArthur } \\ \text { J. Fraser } & \text { OV } & \text { J.C. MacLeod } \\ \text { C.H. Gairns } & \text { CR } & \text { M. Monty Mosher } \\ \text { J.A.F. Gardner } & \text { VA } & \text { J.K. Naysmith } \\ \text { G.J. Garner } & \text { LW } & \text { V.J. Nordin }\end{array}$

SO
MR
MB
CH
VI
NW
OK
VA
OV
CH
OV
VI
NW
OV

H.A. Peacock

OV

I.C.M. Place

D.R. Redmond

OV

OV

F.C. Robinson

SO

J.H.G. Smith

R.J. Steele

W.M. Stiell

A.M.D. Todd

Barry Volkers

Arden Whidden

J.R.M. Williams

J.C. Wright

A. Wynia

VA

VI

AL

CR

VI

NS

$\mathrm{SO}$

RM

SO

The Contribution of our Individual Sustaining Members is Gratefully Acknowledged 